\title{
"TNOs are Cool”: A survey of the trans-Neptunian region
}

\section{Size and surface characteristics of (90377) Sedna and 2010 EK $_{139}$}

\author{
A. Pál ${ }^{1,2}$, C. Kiss ${ }^{1}$, T. G. Müller ${ }^{3}$, P. Santos-Sanz ${ }^{4}$, E. Vilenius ${ }^{3}$, N. Szalai ${ }^{1}$, M. Mommert ${ }^{5}$, E. Lellouch ${ }^{4}$, M. Rengel ${ }^{6}$, \\ P. Hartogh ${ }^{6}$, S. Protopapa ${ }^{7,6}$, J. Stansberry ${ }^{8}$, J.-L. Ortiz ${ }^{9}$, R. Duffard ${ }^{9}$, A. Thirouin ${ }^{9}$, F. Henry ${ }^{4}$, and A. Delsanti ${ }^{4,10}$ \\ ${ }^{1}$ Konkoly Observatory, MTA Research Centre for Astronomy and Earth Sciences, Konkoly-Thege Miklós út 15-17, 1121 Budapest, \\ Hungary \\ e-mail: apal@szofi.net \\ 2 Department of Astronomy, Loránd Eötvös University, Pázmány Péter sétány 1/A, 1117 Budapest, Hungary \\ 3 Max-Planck-Institut für extraterrestrische Physik, Postfach 1312, Giessenbachstr., 85741 Garching, Germany \\ ${ }^{4}$ LESIA-Observatoire de Paris, CNRS, UPMC Univ. Paris 06, Univ. Paris-Diderot, 5 place J. Janssen, 92195 Meudon Cedex, France \\ 5 Deutsches Zentrum für Luft- und Raumfahrt (DLR), Institut für Planetenforschung, Rutherfordstrasse 2, 12489 Berlin, Germany \\ 6 Max-Planck-Institut für Sonnensystemforschung, Max-Planck-Strasse 2, 37191 Katlenburg-Lindau, Germany \\ 7 Department of Astronomy, University of Maryland, College Park, MD 20742-2421, USA \\ 8 Steward Observatory, University of Arizona, 933 North Cherry Avenue, Tucson, AZ 85721, USA \\ 9 Instituto de Astrofísica de Andalucía - CSIC, Apt 3004, 18008 Granada, Spain \\ 10 Laboratoire d'Astrophysique de Marseille, CNRS \& Université de Provence, 38 rue Frédéric Joliot-Curie, \\ 13388 Marseille Cedex 13, France
}

Received 23 January 2012 / Accepted 26 March 2012

\section{ABSTRACT}

\begin{abstract}
We present estimates of the basic physical properties (size and albedo) of (90377) Sedna, a prominent member of the detached transNeptunian object population and the recently discovered scattered disk object $2010 \mathrm{EK}_{139}$, based on the recent observations acquired with the Herschel Space Observatory, within the "TNOs are Cool!" key programme. Our modeling of the thermal measurements shows that both objects have larger albedos and smaller sizes than the previous expectations, thus their surfaces might be covered by ices in a significantly larger fraction. The derived diameter of Sedna and $2010 \mathrm{EK}_{139}$ are $995 \pm 80 \mathrm{~km}$ and $470_{-10}^{+35} \mathrm{~km}$, while the respective geometric albedos are $p_{\mathrm{V}}=0.32 \pm 0.06$ and $0.25_{-0.05}^{+0.02}$. These estimates are based on thermophysical model techniques.
\end{abstract}

Key words. Kuiper belt objects: individual: (90377) Sedna - radiation mechanisms: thermal - techniques: photometric Kuiper belt objects: individual: 2010 EK139

\section{Introduction}

The Herschel Space Observatory ${ }^{1}$ (Pilbratt et al. 2010) allows the detection of thermal radiation from several trans-Neptunian objects (TNOs) at the precision level of $<1 \mathrm{mJy}$. Since the expected fluxes around the peak of the spectral energy distribution (SED) significantly exceed this precision, Herschel provides a great opportunity to characterize TNOs and obtain basic thermophysical information. In this work, we present recent measurements of the prominent objects (90377) Sedna and 2010 EK 139 using the Photodetector Array Camera and Spectrometer instrument (PACS, Poglitsch et al. 2010) on board the Herschel Space Observatory. These observations are part of the "TNOs are Cool!: a survey of the trans-Neptunian region" Open Time Key Program (Müller et al. 2009, 2010; Lellouch et al. 2010; Lim et al. 2010).

Sedna is a prominent member of the detached objects, that is often classified as an inner Oort-cloud object. Until now, no accurate measurements of the diameter and albedo have been available for this object. Both direct imaging (Brown 2008) and

\footnotetext{
${ }^{1}$ Herschel is an ESA space observatory with science instruments provided by European-led Principal Investigator consortia and with important participation from NASA.
}

upper limits to the thermal radiation using the Spitzer Space Telescope (2.4 mJy at $70 \mu \mathrm{m}$, see Stansberry et al. 2008) have yielded an upper limit of $\approx 1670 \mathrm{~km}$ for its diameter (within $97 \%$ confidence).

$2010 \mathrm{EK}_{139}$ has been discovered in 2010 by Sheppard et al. (2011) in the course of a southern Galactic plane survey. Prediscovery observations date back to 2002, allowing for a relatively accurate orbit determination. This places $2010 \mathrm{EK}_{139}$ among the scattered disk objects. $2010 \mathrm{EK}_{139}$ orbits the Sun on an eccentric orbit $(e \approx 0.53)$ and has a perihelion distance of $q \approx 32.5 \mathrm{AU}$. In addition, $2010 \mathrm{EK}_{139}$ is a suspected member of the $2: 7$ resonance group ${ }^{2}$. We note that a more complete sample of SDOs/detached objects observed with Herschel/PACS is presented by Santos-Sanz et al. (2012).

\section{Observations, data reduction, and photometry}

Sedna was observed by Herschel/PACS in two visits: the first started on 2010 August 6, 10:55:17 UTC and a follow-up started on 2010 August 9, 08:11:37 UTC, both taking place during the

\footnotetext{
${ }^{2}$ http://boulder. swri. edu/ buie/kbo/astrom/10EK139. html
} 
Table 1. Summary of Herschel observations of Sedna and $2010 \mathrm{EK}_{139}$.

\begin{tabular}{lccc}
\hline \hline OBSID & Date \& time (UT) & Angle & Filter \\
\hline Sedna & & & \\
1342202227 & $2010-08-06$ 10:55:17 & $70^{\circ}$ & $\mathrm{B} / \mathrm{R}$ \\
1342202228 & $2010-08-06$ 11:19:54 & $110^{\circ}$ & $\mathrm{B} / \mathrm{R}$ \\
1342202229 & $2010-08-06$ 11:44:31 & $70^{\circ}$ & $\mathrm{G} / \mathrm{R}$ \\
1342202230 & $2010-08-06$ 12:09:08 & $110^{\circ}$ & $\mathrm{G} / \mathrm{R}$ \\
1342202306 & $2010-08-0908: 11: 37$ & $70^{\circ}$ & $\mathrm{B} / \mathrm{R}$ \\
1342202307 & $2010-08-0908: 36: 14$ & $110^{\circ}$ & $\mathrm{B} / \mathrm{R}$ \\
1342202308 & $2010-08-0909: 00: 51$ & $70^{\circ}$ & $\mathrm{G} / \mathrm{R}$ \\
1342202309 & $2010-08-0909: 25: 28$ & $110^{\circ}$ & $\mathrm{G} / \mathrm{R}$ \\
\hline $2010 \mathrm{EK}_{139}$ & & & \\
1342211418 & $2010-12-2307: 04: 30$ & $70^{\circ}$ & $\mathrm{B} / \mathrm{R}$ \\
1342211419 & $2010-12-2307: 15: 01$ & $110^{\circ}$ & $\mathrm{B} / \mathrm{R}$ \\
1342211420 & $2010-12-2307: 25: 32$ & $70^{\circ}$ & $\mathrm{G} / \mathrm{R}$ \\
1342211421 & $2010-12-2307: 36: 03$ & $110^{\circ}$ & $\mathrm{G} / \mathrm{R}$ \\
1342211524 & $2010-12-2319: 58: 27$ & $70^{\circ}$ & $\mathrm{B} / \mathrm{R}$ \\
1342211525 & $2010-12-23$ 20:08:58 & $110^{\circ}$ & $\mathrm{B} / \mathrm{R}$ \\
1342211526 & $2010-12-2320: 19: 29$ & $70^{\circ}$ & $\mathrm{G} / \mathrm{R}$ \\
1342211527 & $2010-12-2320: 30: 00$ & $110^{\circ}$ & $\mathrm{G} / \mathrm{R}$ \\
\hline
\end{tabular}

Notes. The columns are: i) observation identifier, ii) date and time, iii) scan angle direction with respect to the detector array, and iv) filter configuration.

Routine Science Phase observation series of the "TNO's are Cool!" key programme (Müller et al. 2009). 2010 EK 139 was also observed by Herschel/PACS in two visits, the first started on 2010 December 23, 07:04:30 UTC, and a follow-up started the same day, 19:58:27 UTC. Herschel/PACS observed Sedna and $2010 \mathrm{EK}_{139}$ for $\approx 3.14$ and $\approx 1.26 \mathrm{~h}$, respectively. For both objects, we used both the blue/red $(70 / 160 \mu \mathrm{m})$ and green/red $(100 / 160 \mu \mathrm{m})$ channel combinations. The actual details of these observations are summarized in Table 1.

Raw observational data were reduced using the Herschel Interactive Processing Environment (HIPE ${ }^{3}$, see also Ott, 2010) and the processing scripts are similar to the ones employed in Mommert et al. (2012), Santos-Sanz et al. (2012), or Vilenius et al. (2012). For each observation, these scripts create a pair of maps, one for the blue or green channel and one for the red channel. The maps have an effective pixel size of 1 '. $1,1^{\prime \prime} 4$, and 2 '. 1 , for the blue, green, and red filters, respectively: these pixel sizes are set to sample the respective point spread functions (PSFs) properly. Data frames were selected by the actual scan speed $\left(10^{\prime \prime} / \mathrm{s} \leq\right.$ speed $\left.\leq 25^{\prime \prime} / \mathrm{s}\right)$ of the spacecraft, which maximized the effective usage of the detector and yielded significantly higher signal-to-noise $(\mathrm{S} / \mathrm{N})$ ratios than the standard setting (approximately 20"/s).

Since the apparent motion of Sedna and 2010 EK $_{139}$ between the two visits (15-35 map pixels, depending on the actual filter) is relatively large compared to the PSF but small compared to the detector size, the location of the target in the first visit can simply be used as a background area on the maps of the second visit and vice versa. Owing to the satellite pointing uncertainty that is about a few arcsec (Poglitsch et al. 2010), we derived the true map-center displacements using the red channel maps - on which the background confusion is the strongest - as follows. By varying the proper motion vector between the two visits, we computed the cross-correlation residuals for each

\footnotetext{
3 Data presented in this paper were analyzed using "HIPE", a joint development by the Herschel Science Ground Segment Consortium, consisting of ESA, the NASA Herschel Science Center, and the HIFI, PACS and SPIRE consortia members, see http://herschel.esac. esa.int/DpHipeContributors.shtml
}

$70 \mu \mathrm{m}$
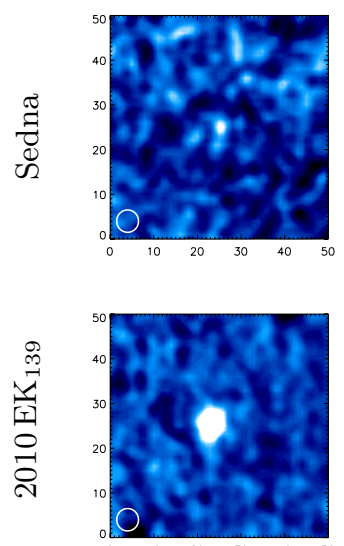

$100 \mu \mathrm{m}$
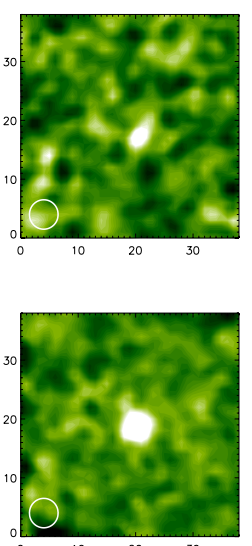

$160 \mu \mathrm{m}$
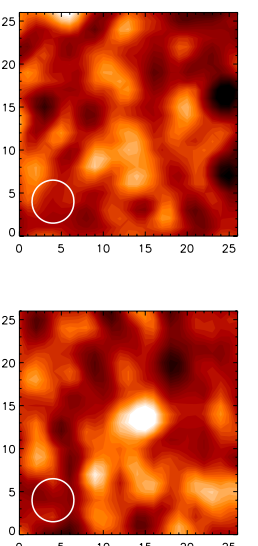

Fig. 1. Image stamps showing the combined maps of Sedna (upper panels) and $2010 \mathrm{EK}_{139}$ (lower panels) in the $70 \mu \mathrm{m}$ (blue), $100 \mu \mathrm{m}$ (green), and $160 \mu \mathrm{m}$ (red) channels. Each stamp covers an area of $56^{\prime \prime} \times 56^{\prime \prime}$, while the tick marks on the axes show the relative positions in pixels. The effective beam size (i.e. the circle with a diameter corresponding to the full width at half magnitude) is also displayed in the lower-left corners of the stamps.

Table 2. Thermal fluxes of Sedna and $2010 \mathrm{EK}_{139}$ derived from our Herschel measurements.

\begin{tabular}{lccc}
\hline \hline Object & Band & $\lambda$ & Flux \\
\hline Sedna & $\mathrm{B}$ & $70 \mu \mathrm{m}$ & $1.8 \pm 0.7 \mathrm{mJy}$ \\
& $\mathrm{G}$ & $100 \mu \mathrm{m}$ & $4.2 \pm 0.9 \mathrm{mJy}$ \\
& $\mathrm{R}$ & $160 \mu \mathrm{m}$ & $2.7 \pm 1.3 \mathrm{mJy}$ \\
\hline $2010 \mathrm{EK}_{139}$ & $\mathrm{~B}$ & $70 \mu \mathrm{m}$ & $17.4 \pm 1.1 \mathrm{mJy}$ \\
& $\mathrm{G}$ & $100 \mu \mathrm{m}$ & $16.3 \pm 1.4 \mathrm{mJy}$ \\
& $\mathrm{R}$ & $160 \mu \mathrm{m}$ & $11.9 \pm 1.8 \mathrm{mJy}$ \\
\hline
\end{tabular}

trial vector. By minimizing the residuals, we obtained a more precise value for the shift between the visits and the photometry of combined maps was found to be more reliable. Since simple averaging the registered maps does not cancel the background confusion noise, we employed background removal techniques as it is described in Santos-Sanz et al. (2012) or Mommert et al. (2012). The maps on which the photometry was then performed are shown in Fig. 1.

Regardless of the background structures, in the subtracted and combined maps the only expected source is the TNO itself and this source can be treated as an isolated point source. We estimated the fluxes and their uncertainties using (1) a single aperture that maximizes the expected $\mathrm{S} / \mathrm{N}$ ratio; (2) the aperture growth curve method and implanted artificial sources in a Monte Carlo fashion (see e.g. Santos-Sanz et al. 2012); and (3) we also checked the individual (non-combined) maps on which they had sufficient $\mathrm{S} / \mathrm{N}$ ratio. For a more detailed description, we refer to Mommert et al. (2012) and Santos-Sanz et al. (2012).

We found that all three methods yielded the same fluxes and uncertainties for each channel. The individual analysis of maps for $2010 \mathrm{EK}_{139}$ also showed consistent results. Therefore, we accepted the means of all measurements per object and filter as final fluxes (see Table 2) and used them for thermal modeling. We note here that the color corrections provided in Poglitsch et al. (2010) are negligible: it is nearly or less than 1 per cent for $2010 \mathrm{EK}_{139}$ and for Sedna, it is 6 per cent in the $70 \mu \mathrm{m}$ channel and less than 3 per cent in the other longer wavelength channels, 
A. Pál et al.: "TNOs are Cool”: A survey of the trans-Neptunian region. VII.
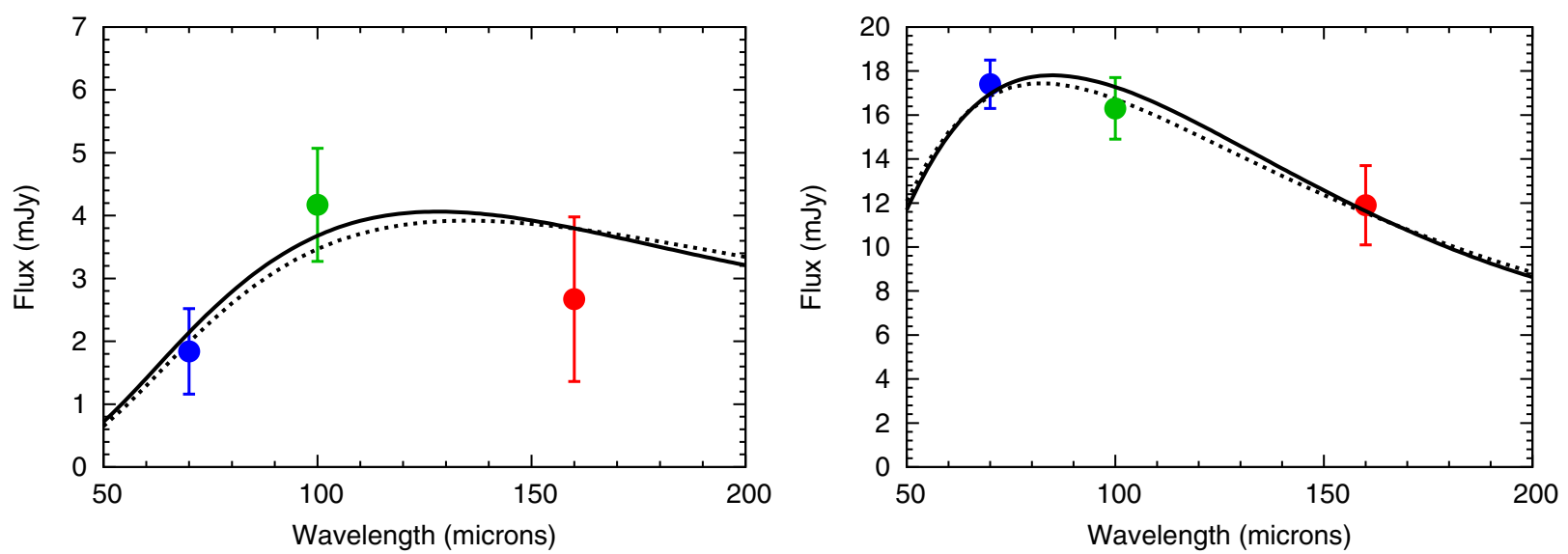

Fig. 2. Spectral energy distribution of Sedna (left) and $2010 \mathrm{EK}_{139}$ (right) in the far-infrared region, based on Herschel/PACS measurements. Superimposed are the best-fit TPM (solid lines) and STM curves with floating beaming parameter (dashed lines).

Table 3. Orbital and optical data for Sedna and $2010 \mathrm{EK}_{139}$ at the time of the Herschel observations.

\begin{tabular}{lcc}
\hline \hline Object & Quantity & Value \\
\hline Sedna & $r$ & $87.43 \mathrm{AU}$ \\
& $\Delta$ & $87.56 \mathrm{AU}$ \\
& $\alpha$ & $0{ }^{\circ}$ \\
& $H_{\mathrm{V}}$ & $+1.83 \pm 0.05$ \\
\hline${\text { 2010 } \text { EK }_{139}}^{r}$ & $39.08 \mathrm{AU}$ \\
& $\Delta$ & $39.50 \mathrm{AU}$ \\
& $\alpha$ & 1.3 \\
& $H_{\mathrm{V}}$ & $+3.80 \pm 0.10$ \\
\hline
\end{tabular}

Notes. The parameters $r$ and $\Delta$ denote the heliocentric distance and the distance from Herschel, $\alpha$ is the phase angle, and $H_{\mathrm{V}}$ is the absolute visual magnitude, which is available from the literature.

so almost less by a magnitude than the relative photometric uncertainties in all of these cases.

\section{Thermal properties}

The basic physical properties of Sedna and 2010 EK $_{139}$ were estimated by a hybrid standard thermal model (STM, Lebofsky et al. 1986; Stansberry et al. 2008) in which the beaming parameter is adjustable and the asteroid thermophysical model (TPM, see Lagerros 1996, 1997, 1998). The absolute magnitudes of the reflected sunlight from these TNOs are available from the literature (Rabinowitz et al. 2007; Sheppard et al. 2011). In addition for $2010 \mathrm{EK}_{139}$, we conservatively increased the formal uncertainty ( $0.03 \mathrm{mag}$, based on MPC data) up to $0.10 \mathrm{mag}$ : we took into account the possible omission of the phase angle corrections and also added quadratically an average TNO lightcurve amplitude of 0.088 mag (based on Duffard et al. 2009). The employed geometric parameters and absolute magnitudes are summarized in Table 3.

The hybrid STM predicts thermal fluxes from the geometric albedo, diameter, and beaming parameter and these fluxes can be computed for arbitrary solar and geocentric distances. Hybrid STM provides reliable estimates only for small phase angles (via a simple form of phase angle corrections), although owing to the distances of these objects, this estimate is fairly sufficient in our cases. To estimate the physical parameters and their respective uncertainties, we used a Monte Carlo (MC) approach by varying the fit input around their mean with the standard deviation equal to their respective uncertainty. For both targets, we used the fixed- $\eta$ approach for the beaming parameter, i.e. taking $\eta=1.14 \pm 0.15$ for each MC step. This mean value of and scatter in the beaming factor are taken from Santos-Sanz et al. (2012) and seem to be an acceptable approach for TNOs. To estimate the phase integral $q$, i.e. the ratio of the Bond to geometric albedo (i.e. $A=q p_{\mathrm{V}}$ ), we employed an iterative approach. First, the phase integral is computed for unity slope parameter $(G=1$, i.e. $q=0.29+0.68 G)$, and then refined using eq. 1 of Brucker et al. (2009) until convergence. This procedure applied for hybrid STM yielded the diameter, geometric albedo, and slope parameter of $D=1060 \pm 100 \mathrm{~km}$, $p_{\mathrm{V}}=0.290 \pm 0.061$, and $G=0.42 \pm 0.04$ for Sedna and $D=535 \pm 30 \mathrm{~km}, p_{\mathrm{V}}=0.187 \pm 0.027$, and $G=0.37 \pm 0.03$ for $2010 \mathrm{EK}_{139}$, respectively. We repeated the similar procedure by allowing the beaming parameter $\eta$ to vary. This analysis yielded $\eta=0.95 \pm 0.43$ for Sedna, with the corresponding diameter and albedo of $D=990 \pm 95 \mathrm{~km}$ and $p_{\mathrm{V}}=0.336 \pm 0.072$. For $2010 \mathrm{EK}_{139}$, the best-fit value of the beaming parameter is somewhat smaller, $0.70 \pm 0.31$, while the diameter and albedo values are $D=450 \pm 35 \mathrm{~km}$ and $p_{\mathrm{V}}=0.261 \pm 0.047$.

In the case of Sedna, we note that the linear phase coefficient $\beta=0.151 \pm 0.033$ (Rabinowitz et al. 2007) would imply a phase integral of $q=0.89_{-0.29}^{+0.55}$, assuming the same phase behavior over the whole phase angle range. Although the phase curve is known for very small domains ( $\alpha \lesssim 0.6^{\circ}$, see also Fig. 2 of Rabinowitz et al. 2007), this value broadly agrees, within a nearly $1-\sigma$ difference from the phase integral of $q=0.59 \pm 0.03$ as implied by the radiometric albedo and the Brucker formula.

The results of the TPM estimates were the following. For Sedna, we used the rotation period of $\approx 10.27 \mathrm{~h}$ (Gaudi et al. 2005) and assumed an equator-on rotation and the most favorable solution for the thermal inertia was found to be $0.2 \mathrm{~J} \mathrm{~m}^{-2} \mathrm{~K}^{-1} \mathrm{~s}^{-1 / 2}$, which corresponds to the diameter of $D=$ $995 \pm 80 \mathrm{~km}$ and the geometric albedo of $p_{\mathrm{V}}=0.32 \pm 0.06$. For $2010 \mathrm{EK}_{139}$, we assumed a period of $12 \mathrm{~h}$ and equator-on rotation and found that this object also requires a very low thermal inertia, $0.1 \mathrm{~J} \mathrm{~m}^{-2} \mathrm{~K}^{-1} \mathrm{~s}^{-1 / 2}$. This may change slightly if the rotation period and the spin vector orientation were very different, although all feasible solutions put the thermal inertia below $1.0 \mathrm{~J} \mathrm{~m}^{-2} \mathrm{~K}^{-1} \mathrm{~s}^{-1 / 2}$. Our best fit yielded a diameter of $D=470_{-10}^{+35} \mathrm{~km}$ and a geometric albedo of $p_{\mathrm{V}}=0.25_{-0.05}^{+0.02}$, which do not differ significantly from the hybrid STM model results in which the beaming parameter was also varied. We note that here the uncertainties include both the statistical errors and the 
ambiguities in the rotation parameters (see also Müller et al. 2011, for more details about this modeling).

In Fig. 2, we displayed the far-infrared SEDs for these two objects as it is estimated from the hybrid STM and TPM fitting and our best-fit data and the floating $\eta$ values. We also note that the rotation period of Sedna found by Gaudi et al. (2005) corresponds to a peak-to-peak amplitude of $0.02 \mathrm{mag}$. The small amplitude does not change the reliability of the thermal modeling and the corresponding shape effects are not relevant to the size determination.

\section{Discussion}

We have estimated the sizes and surface albedos for the transNeptunian objects Sedna and $2010 E_{139}$ using recent observations of their thermal emission at 70/100/160 $\mu \mathrm{m}$ with Herschel/PACS. On the basis of earlier Spitzer measurements, Sedna had already only an upper limit to its size estimate (Stansberry et al. 2008). Our analysis has shown that for Sedna and $2010 \mathrm{EK}_{139}$, the respective geometric albedos are $p_{\mathrm{V}}=$ $0.32 \pm 0.06$ and $p_{\mathrm{V}}=0.25_{-0.05}^{+0.02}$, thus both objects have brighter surfaces than the average TNO population (Stansberry et al. 2008) or SDOs/detached population (Table 5 in Santos-Sanz et al. 2012). We note that the albedos of Sedna and $2010 \mathrm{EK}_{139}$ closely match those of detached objects in Fig. $4 \mathrm{a}\left(p_{\mathrm{V}}\right.$ vs. diameter) in Santos-Sanz et al. (2012). According to Schaller \& Brown (2007) and Brown et al. (2011), even with these newly derived parameters, Sedna lies in the region in which volatiles are expected to be retained in the surface (see Fig. 1 in that paper for an equivalent temperature of $20 \pm 2 \mathrm{~K}$ ), hence one can also expect a brighter surface (see also Barucci et al. 2005; Emery et al. 2007). Sedna is currently approaching its perihelion. Thus, if the brightness of the surface were changing owing to the ongoing sublimation of ices, it might be detectable in the variation in the absolute magnitude on a timescale of decades. In contrast, $2010 \mathrm{EK}_{139}$ falls in the region in which volatiles should have been lost (using $48 \pm 3 \mathrm{~K}$ for equivalent temperature). However, objects of this size can have such a high albedo if water ice is present on the surface (see e.g. Barkume et al. 2006; Ragozzine \& Brown 2009; Dumas et al. 2011). The presence of water could be tested by measuring the intrinsic color to see whether it is bluish (Brown 2008). As they lack known satellites, we do not know the masses and hence the surface gravity and escape velocities of these objects that could place tighter constraints on the surface properties.
Acknowledgements. We gratefully thank the valuable comments and suggestions of the referee, Josh Emery. The work of A.P., Cs.K., and N.Sz. has been supported by the ESA grant PECS 98073. A.P. and Cs.K. were also supported by the János Bolyai Research Scholarship of the Hungarian Academy of Sciences. Cs.K. thanks the support of the OTKA grant K101393. Part of this work was supported by the German DLR projects number 50 OR 1108, 50 OR 0903, and 50 OR 0904. M.R. acknowledges support from the German Deutsches Zentrum für Luft- und Raumfahrt, DLR project number 50OFO 0903. M.M. acknowledges support through the DFG Special Priority Program 1385. P.S.-S. would like to acknowledge financial support by the Centre National de la Recherche Scientifique (CNRS). J.-L.O. acknowledges the Spanish grants AYA2008-06202-C03-01, AYA2011-30106-C02-01 and 2007-FQM2998.

\section{References}

Barkume, K. M., Brown, M. E., \& Schaller, E. L. 2006, ApJ, 640, 87 Barucci, M. A., Cruikshank, D. P., Dotto, E., et al. 2005, A\&A, 439, 1 Brown, M. E. 2008, The Solar System Beyond Neptune, ed. M. A. Barucci, H. Boehnhardt, D. P. Cruikshank, \& A. Morbidelli (Tucson: University of Arizona Press), 592, 335

Brown, M. E., Burgasser, A. J., \& Fraser, W. C. 2011, ApJ, 738, L26

Brucker, M. J., Grundy, W. M., Stansberry, J. A., et al. 2009, Icarus, 201, 284

Duffard, R., Ortiz, J. L., Thirouin, A., Santos-Sanz, P., \& Morales, N. 2009, A\&A, 505, 1283

Dumas, C., Carry, B., Hestroffer, D., \& Merlin, F. 2011, A\&A, 528, A105

Emery, J. P., Dalle Ore, C. M., Cruikshank, D. P., et al. 2007, A\&A, 466, 395

Gaudi, B. S., Stanek, K. Z., Hartman, J. D., Holman, M. J., \& McLeod, B. A. 2005, ApJ, 629, 49

Lagerros, J. S. V. 1996, A\&A, 310, 1011

Lagerros, J. S. V. 1997, A\&A, 325, 1226

Lagerros, J. S. V. 1998, A\&A, 332, 1123

Lebofsky, L. A., Sykes, M. V., Tedesco, E. F., et al. 1986, Icarus, 68, 239

Lellouch, E., Kiss, C., Santos-Sanz, P., et al. 2010, A\&A, 518, A147

Lim, T. L., Stansberry, J., Müller, T. G., et al. 2010, A\&A, 518, A148

Mommert, M., Harris, A. W., Kiss, C., et al. 2012, A\&A, 541, A93

Müller, T. G., Lellouch, E., Böhnhardt, H., et al. 2009, EM\&P, 105, 209

Müller, T. G., Lellouch, E., Stansberry, J., et al. 2010, A\&A, 518, A146

Müller, T. G., D̂rech, J., Hasegawa, S., et al. 2011, A\&A, 525, A145

Ott, S. 2010, in Astronomical Data Analysis Software and Systems XIX, ed. Y. Mizumoto, K.-I. Morita, \& M. Ohishi, ASP Conf. Ser., 434, 139

Pilbratt, G. L., Riedinger, J. R., Passvogel, T., et al. 2010, A\&A, 518, A1

Poglitsch, A., Waelkens, C., Geis, N., et al. 2010, A\&A, 518, A2

Rabinowitz, D. L., Schaefer, B. E., \& Tourtellotte, S. W. 2007, AJ, 133, 26

Ragozzine, D., \& Brown, M. E. 2009, AJ, 137, 4766

Santos-Sanz, P., Lellouch, E., Fornasier, S. et al. 2012, A\&A, 541, A92

Schaller, E. L., \& Brown, M. E. 2007, ApJ, 659, 61

Sheppard, S. S., Udalski, A., Trujillo, C., et al. 2011, AJ, 142, 98

Stansberry, J., Grundy, W., Brown, M., et al. 2008, The Solar System Beyond Neptune, ed. M. A. Barucci, H. Boehnhardt, D. P. Cruikshank, \& A. Morbidelli (Tucson: University of Arizona Press), 592, 161

Vilenius, E., Kiss, C., Mommert, M., et al. 2012, A\&A, 541, A94 